\title{
Modelo difuso para la evaluación de condiciones apropiadas para turismo en litorales de México
}

\author{
César A. Hoil-Rosas, José J. Carbajal-Hernández, Luis P. Sánchez-Fernández, \\ Alberto D. Dávila-Lamas \\ Instituto Politécnico Nacional, Centro de Investigación en Computación, \\ Ciudad de México, México \\ cesarhoil_92@hotmail.com, \{jcarbajalh, lsanchez\}@cic.ipn.mx, \\ addavlamegmail.com
}

\begin{abstract}
Resumen. En la actualidad, el turismo representa una de las actividades económicas de mayor importancia para México, debido a los ingresos anuales que este produce. Sin embargo, la falta de tecnología aplicada en este campo evita el crecimiento del turismo debido a la falta de información de las zonas costeras. En este trabajo, se desarrolla un modelo computacional basado en un Proceso de Jerarquía Analítica por sus siglas en inglés (AHP), con la finalidad de obtener un índice de evaluación de costas. Para este modelo se utilizan parámetros como la temperatura, oleaje, radiación solar, humedad y velocidad del viento, los cuales fueron medidos y analizados. Este índice ayuda a proporcionar información relevante para los turistas que se disponen a viajar a México.
\end{abstract}

Palabras clave: AHP, turismo, costas, procesamiento de señales, evaluación.

\section{Diffuse Model for the Evaluation of Appropriate Conditions for Tourism in the Mexican Coastlines}

\begin{abstract}
Currently, tourism represents one of the most important economic activities for Mexico, due to the annual income it produces. However, the lack of technology applied in this field prevents the growth of tourism due to the lack of information on coastal areas. In this work, a computational model based on an Analytical Hierarchy Process (AHP) is developed, with the purpose of obtaining a cost evaluation index. Parameters such as temperature, waves, solar radiation, humidity and wind speed are used for this model, which were measured and analyzed. This index helps to provide relevant information for tourists who are preparing to travel to Mexico.
\end{abstract}

Keywords: AHP, tourism, coasts, signal processing, evaluation.

\section{Introducción}

El turismo es una actividad en expansión en el mundo con un crecimiento anual en el número de viajeros y recursos económicos que produce esta actividad [1]. En los 
últimos años el turismo ha adquirido una importancia relevante como generador de ingresos y como fuente de empleo, en especial para las economías emergentes a nivel mundial. Según la OMT [2], las llegadas internacionales anuales superan los 800 millones en todo el mundo, generando ingresos de aproximadamente 800 billones de dólares por año.

En este aspecto, el turismo en México es una de las actividades económicas más relevantes del país y es una de las más importantes en el mundo, ocupando el sexto lugar a nivel mundial en llegadas de turistas internacionales con 42 millones de visitantes en 2018 [3], lo cual contribuyó con el $8.2 \%$ del PIB nacional; cabe mencionar que el $45 \%$ de esta actividad está orientada a la zona costera [4].

A lo largo de la historia el turismo ha impactado sobre todo lo que ha tenido contacto, hoy en día las ciudades enfrentan retos complejos al lidiar con ella como el aumento de la población mundial y la transformación digital. Ante esta situación las ciudades y sus gobernantes deben diseñar nuevas estrategias para mejorar aspectos como la planificación y gestión de sus territorios, con la finalidad de garantizar un desarrollo sostenible, al minimizar efectos negativos en el medioambiente, fomentar el bienestar económico y elevar la calidad de vida de los pobladores.

En la literatura se pueden encontrar proyectos en los cuales se realizan clasificaciones de zonas turísticas de acuerdo con determinadas variables como son la urbanización, el riesgo, entre otras. Ranieri en 2016 [5] analiza las condiciones oceanográficas en las playas de Salinópolis y actividades relacionadas con el ocio y la ocupación humana, con el objetivo de categorizar en términos de urbanización. En otro estudio, Irene en 2010 [6] presenta un artículo en el cual se realiza la evaluación subjetiva de los riesgos ambientales asociados con un puerto turístico al sur de Italia, comparando los resultados para proporcionar estimaciones de seguridad ambiental. Estos trabajos analizan y clasifican de acuerdo con datos obtenidos de manera subjetiva por lo cual los resultados se ven afectados por la percepción de cada autor.

Por otro lado, diversos investigadores se han enfocado en la evaluación del paisaje en las zonas costeras. En Brasil, Cristiano (2016) [7], evaluó el paisaje costero del municipio de Torres, tomando en cuenta 26 parámetros naturales y humanos para clasificar las playas en 5 clases, siendo la clase 1 la de mayor atractivo paisajístico y la clase 5 la menos atractiva. Los resultados pusieron de manifiesto una mala gestión de las zonas costeras, con gran cantidad de basura en las playas, instalaciones de apoyo turístico situadas en lugares inadecuados y vertidos de aguas residuales. En 2004, Ergin [8] utilizó 26 parámetros para evaluar los paisajes de las costas en Turquía y Reino Unido, a través de encuestas a turistas y expertos en percepción del paisaje. Después para el año 2010 [9], realizó la evaluación de 34 zonas costeras en la región occidental del Mar Negro.

Los trabajos anteriores presentan un gran avance en el tema de las evaluaciones de zonas costeras. Sin embargo, ninguno está enfocado en crear una herramienta que tome en consideración las prioridades que tiene cada visitante para ayudar al crecimiento del turismo. Además, la falta de tecnología empleada para realizar las mediciones de los diferentes parámetros involucrados evita realizar evaluaciones objetivas.

Con el propósito de establecer las condiciones apropiadas para el turismo en los litorales de México, el presente proyecto propone la creación de un índice de evaluación de costas utilizando un modelo de prioridades basado en un Proceso de Jerarquía Analítica (AHP) y el procesamiento de parámetros climáticos y oceanológicos. Este 
índice tiene como principal ventaja el adaptarse a las necesidades de los diferentes turistas que visitan una misma zona ya que permitirá proporcionar información necesaria para los visitantes que se disponen de viajar a las playas de México, permitiendo encontrar los periodos adecuados y los sitios que sean de su mayor interés.

\section{Requerimientos}

Hoy en día existen diversos criterios para establecer las actividades que se pueden realizar en una zona determinada, los cuales se basan en las condiciones ideales de un conjunto de parámetros. Debido a esto, la siguiente sección explica las condiciones necesarias para llevar a cabo determinadas actividades en las zonas costeras.

\subsection{Análisis de factores}

Normalmente, los factores de análisis son de gran relevancia para solucionar un problema y estos a su vez constan de varios parámetros que definen su importancia. En este proyecto se consideran 4 factores principales. El primero es la recreación familiar, debido a que ciertas playas no cuentan con las condiciones necesarias para ser visitadas por familias. El segundo son las playas vírgenes, este factor se refiere a la cantidad de infraestructura disponible en el lugar y que facilita las necesidades básicas de los turistas (hotelería, sanitarios, comunicaciones, etc.). El siguiente es la seguridad, el cual es fundamental para brindar protección a turistas que desconocen la zona. Finalmente, la realización de actividades acuáticas, el cual proporciona un nivel de diversión deseada al practicar actividades como el surf, buceo, pesca, etc. De esta forma los factores lo podemos identificar de la siguiente manera:

- F1: Recreación Familiar,

- F2: Playas vírgenes,

- F3: Seguridad,

- F4: Actividades acuáticas

\subsection{Análisis de parámetros}

El primer análisis está basado en los parámetros que integran cada factor. Como se mencionó con anterioridad, estos tienen diferente importancia dependiendo de las necesidades del turista ya que no es la misma percepción de aquellos que viajan en familia a lo que prefieren sitios escondidos en lugares vírgenes. En la Tabla 1, se muestran una clasificación de algunos parámetros con base en aquellos que pueden ser medidos con sensores y otros que solo pueden ser evaluados mediante encuestas.

En este trabajo, el análisis de los parámetros que pueden ser automatizados es un conjunto de estudio, ya que puede obtenerse información sobre ellos y establecer las reglas sobre los niveles máximos o mínimos que deben de presentar para obtener un nivel tanto de seguridad como de aceptación del turista. En la Tabla 2, se presenta la clasificación de los parámetros con base en su rango óptimo, así como las tolerancias para su medición. Estos valores fueron obtenidos de otros trabajos de investigación especializados en el turismo [10-12]. 
César A. Hoil-Rosas, José J. Carbajal-Hernández, Luis P. Sánchez-Fernández, et al.

Tabla 1. Tipos de parámetros involucrados en el estudio de preferencias.

\begin{tabular}{|c|c|c|c|}
\hline & Institucional & Automatizado & Encuesta \\
\hline - & Crimen & Temperatura & $\begin{array}{ll} & \text { Color del agua }\end{array}$ \\
\hline - & Guardavidas & Oleaje & - $\quad$ Tipo de arena \\
\hline- & Contaminación & Radiación solar & - $\quad$ Paisaje \\
\hline 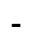 & Venta informal & - $\quad$ Humedad & - $\quad$ Tipo de vegetación \\
\hline- & Mantenimiento & - $\quad$ Velocidad del viento & - $\quad$ Atardecer \\
\hline
\end{tabular}

Tabla 2. Valores óptimos y límites difusos de cada parámetro.

\begin{tabular}{|c|c|c|c|c|c|c|}
\hline \multirow{2}{*}{ Parámetro } & \multirow{2}{*}{ Rango Óptimo } & \multirow{2}{*}{ Unidades } & \multicolumn{4}{|c|}{ Límites tolerables } \\
\hline & & & $a$ & $b$ & $c$ & $d$ \\
\hline Temperatura $(t)$ & $25-30$ & ${ }^{\circ} \mathrm{C}$ & 22 & 25 & 30 & 33 \\
\hline Oleaje $(o)$ & $0.5-2$ & $m$ & 0 & 0.5 & 2 & 3 \\
\hline Radiación solar $(r)$ & $6.1-10.1$ & $\mathrm{~kW} / \mathrm{m}^{2}$ & 6.1 & 8.1 & 10.1 & 0 \\
\hline Humedad $(h)$ & $40-70$ & $\%$ & 40 & 55 & 70 & 0 \\
\hline Velocidad del viento $(v)$ & $4-12$ & $\mathrm{Km} / \mathrm{hr}$ & 1 & 4 & 12 & 16 \\
\hline
\end{tabular}

\section{Modelo de evaluación}

\subsection{Entradas difusas}

Para realizar el análisis de cada parámetro, se propone la evaluación previa mediante una función difusa, misma que permita normalizar los niveles de medición de cada parámetro en un rango de [0-1]. Con el fin de evaluar cada parámetro, se han desarrollado las siguientes funciones difusas, tomando en cuenta los límites tolerables $(a, b, c, d)$ establecidos en la Tabla 2:

$$
\begin{gathered}
\mu(t)=\max \left\{\left(\frac{x-22}{25-22}, 1, \frac{33-x}{33-30}\right), 0\right\}, \\
\mu(o)=\max \left\{\left(\frac{x-0}{0.5-0}, 1, \frac{3-x}{3-2}\right), 0\right\}, \\
\mu(r)=\max \left\{\left(\frac{x-6.1}{8.1-6.1}, 1, \frac{10.1-x}{10.1-8.1}\right), 0\right\}, \\
\mu(h)=\max \left\{\left(\frac{x-40}{55-40}, 1, \frac{70-x}{70-40}\right), 0\right\}, \\
\mu(v)=\max \left\{\left(\frac{x-1}{4-1}, 1, \frac{16-x}{16-12}\right), 0\right\} .
\end{gathered}
$$

\subsection{Proceso analítico jerárquico (AHP)}

En este proyecto se utiliza el modelo AHP propuesto por Saaty [13], para realizar la evaluación de los litorales turísticos. Esta metodología asigna un nivel de importancia 
a cada parámetro dependiendo del factor que se esté evaluando, la interpretación de las escalas se muestra en la Tabla 3.

Tabla 3. Escala propuesta por Saaty en 1980 [14].

\begin{tabular}{|c|c|c|c|c|c|c|c|c|c|}
\hline \multirow{6}{*}{ Importancia } & \multirow{4}{*}{$\begin{array}{l}\text { Ig } \\
\text { ua }\end{array}$} & \multirow[b]{4}{*}{+} & \multirow{2}{*}{ M } & \multirow[b]{4}{*}{+} & \multicolumn{3}{|r|}{$\mathrm{M}$} & & $\Gamma$ \\
\hline & & & & & $\mathrm{F}$ & & uy & & $\mathrm{E}$ \\
\hline & & & od & & ue & & $\mathrm{F}$ & & $\mathrm{Xl}$ \\
\hline & & & er & & 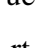 & + & & + & re \\
\hline & 1 & & $\mathrm{ad}$ & & $\pi$ & & ue & & $\mathrm{m}$ \\
\hline & & & o & & $\mathrm{e}$ & & $\mathrm{rt}$ & & o \\
\hline Escala & 1 & 2 & 3 & 4 & 5 & 6 & 7 & 8 & 9 \\
\hline
\end{tabular}

Posteriormente, se crea una matriz pareada $\left(A=a_{i j}\right)$ donde las prioridades de importancia dadas pueden ser asignadas entre parámetros $\left(\frac{w_{a}}{w_{b}}\right)$. Esta matriz es cuadrada, recíproca y positiva; y se construye como se muestra a continuación:

$$
\begin{aligned}
& A=P_{1} \quad P_{2} \cdots P_{n} \quad P_{1} P_{2}
\end{aligned}
$$

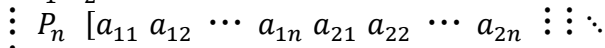

$$
\begin{aligned}
& \left.\vdots a_{n 1} a_{n 2} \cdots a_{n n}\right] \\
& =\left[\frac{w_{1}}{w_{1}} \frac{w_{1}}{w_{2}} \cdots \frac{w_{1}}{w_{n}} \frac{w_{2}}{w_{1}} \frac{w_{2}}{w_{2}} \cdots \frac{w_{2}}{w_{n}}: \vdots \vdots \vdots \frac{w_{n}}{w_{1}} \frac{w_{n}}{w_{2}} \cdots \frac{w_{n}}{w_{n}}\right] \text {. }
\end{aligned}
$$

Una vez elaborada la matriz, se procede a calcular lo que se denomina como eigenvector [15], el cual ayudará a determinar la prioridad de los pesos. Lo cuales serán usados para calcular el índice de evaluación de costas. Con base en lo anterior, la matriz cuadrada $\mathbf{B}$ se calcula de la siguiente manera:

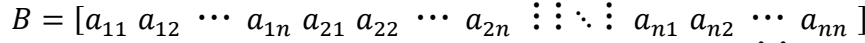

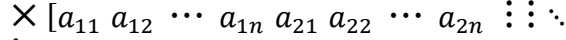

$$
\begin{aligned}
& \left.\vdots a_{n 1} a_{n 2} \cdots a_{n n}\right] \text {. }
\end{aligned}
$$

La sumatoria de las filas es determinada de acuerdo con:

$$
C_{i}=\sum_{j=1}^{n} \quad B_{i j} \forall i=1,2, \ldots, n .
$$

Finalmente, la importancia de los pesos es obtenida normalizando el vector $C_{i}$, como se muestra en la siguiente fórmula:

$$
W_{i}=\frac{C_{i}}{\sum_{j=1}^{n} C_{j}} \forall i=1,2, \ldots, n
$$

Una consideración importante que ofrece el método de AHP es medir el grado de consistencia de la matriz de comparaciones pareadas. Si el grado de consistencia es aceptable, puede continuarse con el proceso de decisión y si es inaceptable, se debe rehacer la matriz. Este grado puede calcularse por medio de la siguiente fórmula: 


$$
C R=\frac{\lambda_{\max }-n}{(n-1) R I}
$$

donde $\lambda_{\max }$ es el máximo eigenvalor de la matriz pareada, $n$ es el tamaño de la matriz, $R I$ es el índice de consistencia aleatoria y $C R$ es el radio de consistencia. El valor de $R I$ depende del número de elementos que se compara, tal y como se muestra en la Tabla 4 [16].

Tabla 4. Índice de consistencia aleatoria.

\begin{tabular}{ccccccccccc}
\hline $\begin{array}{c}\text { Tamaño de la } \\
\text { matriz }\end{array}$ & 1 & 2 & 3 & 4 & 5 & 6 & 7 & 8 & 9 & 10 \\
\hline$R I$ & 0 & 0 & 0.52 & 0.89 & 1.11 & 1.25 & 1.35 & 1.4 & 1.45 & 1.49 \\
\hline
\end{tabular}

El resultado obtenido por $C R$ nos indica que, si este excede de 0.10 se tiene una matriz inconsistente, por el contrario, si este es menor o igual a 0.10 , la matriz tiene un grado de consistencia aceptable.

\section{3. Índice de evaluación de costas}

El Índice de Evaluación de Costas $(I E C)$ es un valor difuso en un rango de [0-1], el cual se ve afectado de manera negativa por la importancia del peso de cada parámetro, generando de esta forma un puntaje que nos indica que tan buena o mala es la evaluación de la playa para realizar alguna actividad turística. El índice puede describirse de forma matemática por medio de la siguiente fórmula:

$$
I E C=\sum_{i=1}^{n} \mu_{i}(k) W_{i}^{k},
$$

donde $n$ es la cantidad de parámetros, $\mu_{i}(k)$ es el valor difuso obtenido de las ecuaciones definidas en la sección 3.1; $k$ refiere al parámetro y $W$ es la importancia del peso calculado por el método de AHP. Reemplazando cada parámetro en la ecuación 11, se obtiene la siguiente expresión:

$$
I E C=\mu(t) W^{t}+\mu(o) W^{o}+\mu(r) W^{r}+\mu(h) W^{h}+\mu(v) W^{v},
$$

donde $t$ se refiere a la temperatura, $o$ al oleaje, $r$ a la radiación solar, $h$ a la humedad y $v$ a la velocidad del viento. Finalmente, el resultado obtenido será el índice de evaluación de costas, donde mientras más cercano esté a "1" será más recomendable el lugar y por el contrario mientras más próximo esté a " 0 " menos recomendable será el lugar.

\section{Resultados y discusiones}

Para comprobar la eficiencia del índice de evaluación de costas, se realizó una prueba ejemplificando un viaje familiar, asignando la importancia a los parámetros y factores, que resultan de mayor relevancia en este tipo de viajes. En este caso, las prioridades de los factores elegidos de mayor a menor importancia son la seguridad, recreación familiar, actividades acuáticas y playas vírgenes; mientras que los parámetros más 
relevantes para ellos son el oleaje, la radiación solar, la velocidad del viento, la temperatura y la humedad. El procedimiento y los resultados se muestran a continuación.

\subsection{Análisis de comparaciones}

De acuerdo con el orden establecido por la familia en la sección anterior se establecen las matrices de comparaciones. La Tabla 5 muestra las comparaciones entre factores, definidos con base en los criterios de una familia. También, se muestra una comparación entre el método de normalización y el AHP (Eigenvector) siendo este último más preciso. Las tablas 6, 7, 8 y 9 tienen las respectivas matrices pareadas para cada parámetro, donde las comparaciones se realizan teniendo en cuenta el factor como criterio de decisión.

Tabla 5. Comparación de los Factores con un $\mathrm{CR}=0.0348$.

\begin{tabular}{lcccccc}
\hline \multicolumn{1}{c}{ Factores } & F1 & F2 & F3 & F4 & Normalizado & Eigenvector \\
\hline F1: Recreación familiar & 1 & 5 & $1 / 3$ & 4 & 0.2990 & 0.2669 \\
F2: Playas vírgenes & $1 / 5$ & 1 & $1 / 9$ & $1 /$ & 0.0524 & 0.0533 \\
F3: Seguridad & 3 & 9 & 1 & 6 & 0.5497 & 0.5911 \\
F4: Actividades acuáticas & $1 / 4$ & 2 & $1 / 6$ & 1 & 0.0989 & 0.0887 \\
\hline & & & & Suma: & 1 & 1 \\
\hline
\end{tabular}

Tabla 6. Comparación de los parámetros de acuerdo con $\mathrm{F} 1$ y un $C R=0.085$.

\begin{tabular}{lccccccc}
\hline \multicolumn{1}{c}{ Parámetros } & $t$ & $o$ & $r$ & $h$ & $v$ & Normalizado & Eigenvector \\
\hline (t) Temperatura & 1 & 4 & $1 / 3$ & 6 & 7 & 0.314 & 0.286 \\
(o) Oleaje & $1 / 4$ & 1 & $1 / 5$ & 3 & 5 & 0.1619 & 0.1197 \\
(r) Radiación & 3 & 5 & 1 & 7 & 9 & 0.4282 & 0.5055 \\
(h) Humedad & $1 / 6$ & $1 / 3$ & $1 / 7$ & 1 & 2 & 0.0624 & 0.054 \\
(v) Viento & $1 / 7$ & $1 / 5$ & $1 / 9$ & $1 / 2$ & 1 & 0.0335 & 0.0348 \\
\hline
\end{tabular}

Tabla 7. Comparación de los parámetros de acuerdo con $\mathrm{F} 2$ y un $C R=0.0665$.

\begin{tabular}{lccccccc}
\hline \multicolumn{1}{c}{ Parámetros } & $t$ & $o$ & $r$ & $h$ & $v$ & Normalizado & Eigenvector \\
\hline (t) Temperatura & 1 & 3 & $1 / 5$ & $1 / 3$ & 2 & 0.126 & 0.1113 \\
(o) Oleaje & $1 / 3$ & 1 & $1 / 9$ & $1 / 6$ & 2 & 0.0696 & 0.0578 \\
(r) Radiación & 5 & 9 & 1 & 3 & 7 & 0.482 & 0.5245 \\
(h) Humedad & 3 & 6 & $1 / 3$ & 1 & 4 & 0.2763 & 0.2535 \\
(v) Viento & $1 / 2$ & $1 / 2$ & $1 / 7$ & $1 / 4$ & 1 & 0.0461 & 0.0529 \\
\hline
\end{tabular}


César A. Hoil-Rosas, José J. Carbajal-Hernández, Luis P. Sánchez-Fernández, et al.

Tabla 8. Comparación de los parámetros de acuerdo con F3 y un $C R=0.0841$.

\begin{tabular}{lccccccc}
\hline \multicolumn{1}{c}{ Parámetros } & $t$ & $o$ & $r$ & $h$ & $v$ & Normalizado & Eigenvector \\
\hline (t) Temperatura & 1 & $1 / 7$ & $1 / 5$ & 3 & $1 / 3$ & 0.0816 & 0.0634 \\
(o) Oleaje & 7 & 1 & 3 & 9 & 5 & 0.4361 & 0.5128 \\
(r) Radiación & 5 & $1 / 3$ & 1 & 7 & 3 & 0.2849 & 0.2615 \\
(h) Humedad & $1 / 3$ & $1 / 9$ & $1 / 7$ & 1 & $1 / 5$ & 0.0312 & 0.0333 \\
(v) Viento & 3 & $1 / 5$ & $1 / 3$ & 5 & 1 & 0.1662 & 0.1290 \\
\hline
\end{tabular}

Tabla 9. Comparación de los parámetros de acuerdo con F4 y un $C R=0.05975$.

\begin{tabular}{lccccccc}
\hline \multicolumn{1}{c}{ Parámetros } & $t$ & $o$ & $r$ & $h$ & $v$ & Normalizado & Eigenvector \\
\hline (t) Temperatura & 1 & $1 / 5$ & 4 & 4 & $1 / 3$ & 0.1705 & 0.1310 \\
(o) Oleaje & 5 & 1 & 8 & 8 & 3 & 0.4468 & 0.5167 \\
(r) Radiación & $1 / 4$ & $1 / 8$ & 1 & 1 & $1 / 6$ & 0.0454 & 0.0445 \\
(h) Humedad & $1 / 4$ & $1 / 8$ & 1 & 1 & $1 / 6$ & 0.0454 & 0.0445 \\
(v) Viento & 3 & $1 / 3$ & 6 & 6 & 1 & 0.2919 & 0.2633 \\
\hline
\end{tabular}

Para obtener los pesos finales de cada parámetro es necesario multiplicar los pesos obtenidos en la Tabla 5 por los pesos individuales de cada parámetro con respecto a cada factor. La Tabla 10 muestra los valores finales obtenidos por el método de AHP.

Tabla 10. Comparación de los parámetros considerando todos los factores y pesos.

\begin{tabular}{|c|c|c|c|c|c|c|c|c|c|c|c|}
\hline Paráme & $t$ & $o$ & $r$ & $h$ & $v$ & $\begin{array}{l}\text { Pesos de } \\
\text { factores }\end{array}$ & $t$ & $o$ & $r$ & $h$ & $v$ \\
\hline $\mathrm{F} 1$ & 0.2860 & 0.1197 & 0.5055 & 0.054 & 0.0348 & 0.2669 & 0.0763 & 0.0319 & 0.1349 & 0.0144 & 0.0093 \\
\hline $\mathrm{F} 2$ & 0.1113 & 0.0578 & 0.5245 & 0.2535 & 0.0529 & 0.0533 & 0.0059 & 0.0031 & 0.0280 & 0.0135 & 0.0028 \\
\hline F3 & 0.0634 & 0.5128 & 0.2615 & 0.0333 & 0.1290 & 0.5911 & 0.0375 & 0.3031 & 0.1546 & 0.0197 & 0.0763 \\
\hline F4 & 0.1310 & 0.5167 & 0.0445 & 0.0445 & 0.2633 & 0.0887 & 0.0116 & 0.0458 & 0.0039 & 0.0039 & 0.0234 \\
\hline \multicolumn{2}{|c|}{ Score Final } & & & & & 1 & 0.1314 & 0.3840 & 0.3214 & 0.0516 & 0.1117 \\
\hline
\end{tabular}

\subsection{Medición de parámetros}

Las mediciones de los parámetros fueron realizadas durante los días del 2 de junio al 12 de junio del 2019 en la ciudad de La Paz, Baja California Sur. La base de datos se formó al medir los parámetros cada 15 minutos durante 11 días, obteniendo 96 muestras diarias y un total de 1056 muestras por cada uno. Los parámetros medidos son la temperatura, el oleaje, la radiación solar, la humedad y la velocidad del viento. La Figura 1, muestra gráficamente el comportamiento de cada uno de estos, así como los límites permitidos que se establecieron en la Tabla 2. 
Modelo difuso para la evaluación de condiciones apropiadas para turismo en litorales de México
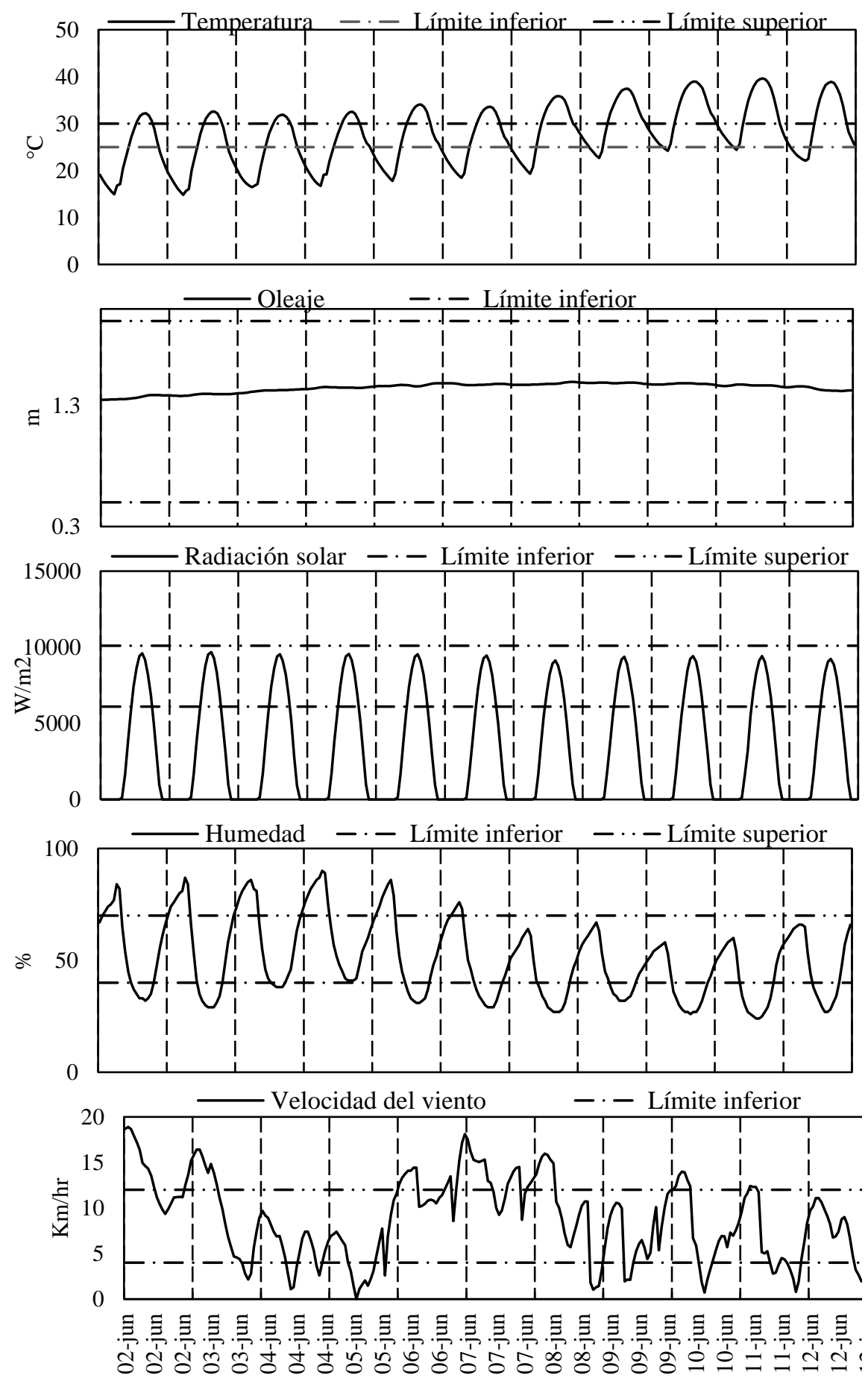

Fig. 1. Gráficas de cada parámetro durante los días del 2 al 12 de junio de 2019. 
En la Figura 1, se pueden apreciar un comportamiento aleatorio en cada parámetro, los cuales sobrepasan los límites óptimos establecidos para cada uno; a excepción del oleaje el cual siempre se mantiene dentro del rango óptimo.

\section{3. Índice de medición de costas}

Finalmente, para obtener el Índice de Medición de Costas se utiliza la ecuación 12, tomando en cuenta los pesos obtenidos en la Tabla 10, así como el resultado de la evaluación de cada parámetro de acuerdo a las funciones difusas establecidas en las ecuaciones 1-5.

En la Figura 2 se muestra el resultado de la evaluación de la costa de la Paz, Baja California Sur. Como se observa, tuvo una evaluación regular debido a que la mayoría de los parámetros estaban fuera del rango permitido. Sin embargo, como se muestra en la Tabla 10 el oleaje es el que tiene mayor peso y esto hace que el índice sea regular ya que este parámetro siempre se mantuvo en el rango permitido. Analizando la gráfica por día, observamos que la mejor evaluación se da en los días del 9 al 12 de junio de 2019 , esto se debe a que la humedad y la velocidad del viento, permanecieron en mayor medida en su rango óptimo; por lo cual el factor principal establecido en la sección 4 por la familia, que es la seguridad, es mejor evaluada, obteniendo así una mayor calificación en el índice.

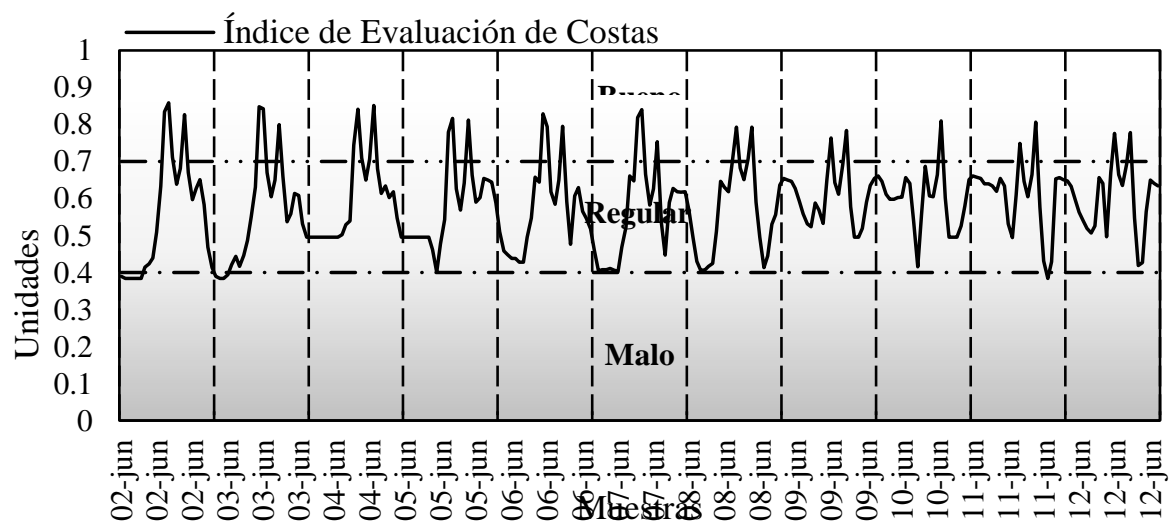

Fig. 2. Gráfica del índice de evaluación de costas para La Paz, Baja California Sur.

\section{Conclusión y trabajo a futuro}

El índice de evaluación de costas presente en este trabajo es una herramienta novedosa ya que es adaptativa a las necesidades de cada turista y a los parámetros presentes en cada costa. Este índice ayuda a los turistas a tener una mayor información de la playa a visitar, que les ayudará a encontrar el periodo y la costa que mejor se adapte a sus necesidades.

De acuerdo con la literatura, existen trabajos similares que se encargan de evaluar diferentes características de una playa; sin embargo, nos están enfocados en ayudar al turismo, por lo cual este modelo es innovador ya que conjunta todos los factores que el turista necesita para indicarle si las condiciones en playa son buena o mala de acuerdo 
con sus preferencias. Como trabajo a futuro, se plantea el aumentar el número de parámetros a medir, integrando otras técnicas y modelos computacionales para obtener un mejor análisis y evaluación de las costas.

\section{Referencias}

1. Macário de Oliveira, V., Pasa Gómez, C., Ataíde Cândido, G.: Indicadores de sustentabilidad para la actividad turística. Una propuesta de monitoreo usando criterios de análisis. Estudios y perspectivas en turismo 22(2) (2013)

2. OMT-Organización Mundial del Turismo: Tourism highlights 2006 Edition. Madrid (2006)

3. SECTUR: Visión Global del Turismo a México. Análisis de mercados, perspectivas del turismo mundial (2018)

4. SECTUR: Turismo de internación 2001-2005, Visitantes internacionales hacia México (2006)

5. Ranieri, L.A., El-Robrini, M.: Condição Oceanográfica, Uso e Ocupação da Costa de Salinópolis (Setor Corvina-Atalaia), Nordeste do Pará, Brasil. Revista de Gestão Costeira Integrada 16(2), 133-146 (2016)

6. Irene, P., Paolo, V., Donatella, V., Alberto, M.J., Mauro, F., Giovanni, Z.: Mapping the environmental risk of a tourist harbor in order to foster environmental security: objective vs. subjective assessments. Marine pollution bulletin 60(7), 1051-1058 (2010)

7. Cristiano, S.D.C., Rockett, G.C., Portz, L.C., Anfuso, G., Gruber, N.L.S., Williams, A.T.: Evaluation of Coastal Scenery in Urban Beach: Torres, Rio Grande do Sul, Brazil. Revista Gestão Costeira Integrada Journal of Integrated Coastal Zone Management. Lisboa: Associação Portuguesa dos Recursos Hídricos, Vol. 16, n. 1, pp. 71-78 (2016)

8. Ergin, A., Karaesmen, E., Micallef, A., Williams, A.T.: A new methodology for evaluating coastal scenery: fuzzy logic systems. Area, 36(4), 367-386 (2004)

9. Ergin, A., Özölçer, İ.H., Şahin, F.: Evaluating coastal scenery using fuzzy logic: Application at selected sites in Western Black Sea coastal region of Turkey. Ocean Engineering 37(7), 583-591 (2010)

10. Kim, D.I., Matsuyama, Y., Nagasoe, S., Yamaguchi, M., Yoon, Y.H., Oshima, Y., Honjo, T.: Effects of temperature, salinity and irradiance on the growth of the harmful red tide dinoflagellate Cochlodinium polykrikoides Margalef (Dinophyceae). Journal of Plankton Research 26(1), 61-66 (2004)

11. Gilmore, J.W.: Cálculo y mapeo de la radiación solar directa y difusa en Costa Rica. Uniciencia 22(1), 55-69 (2008)

12. Cengel, Y.A., Boles, M.A., Buesa, I.A.: Termodinámica, vol. 10. São Paulo: McGrawHill (2006)

13. Saaty, T.L.: Decision making with the analytic hierarchy process. International journal of services sciences 1(1), 83-98 (2008)

14. Saaty, T.L., Wind, Y.: Marketing applications of the analytic hierarchy process. Management science 26(7), 641-658 (1980)

15. Perron, O.: Grundlagen für eine Theorie des Jacobischen Kettenbruchalgorithmus. Math. Ann. 64, 11-76 (1907)

16. Toskano, G., Bruno, G.: El proceso de análisis jerárquico (AHP) como herramienta para la toma de decisiones en la selección de proveedores. Trabajo de grado, Universidad Nacional Mayor de San Marcos, Lima, Perú (2005) 